\title{
Disorder and superconductivity : a new phase of bi-particle localized states
}

\author{
Bhargavi Srinivasan and Dima L. Shepelyansky \\ Laboratoire de Physique Quantique, UMR 5626 du CNRS, Université Paul Sabatier, F-31062 Toulouse Cedex 4, France
}

(November 23, 2018)

\begin{abstract}
We study the two-dimensional, disordered, attractive Hubbard model by the projector quantum Monte Carlo method and Bogoliubov - de Gennes mean-field theory. Our results for the ground state show the appearance of a new phase with charge localization in the metallic regime of the non-interacting model. Contrary to the common lore, we demonstrate that mean-field theory fails to predict this phase and is unable to describe the correct physical picture in this regime.
\end{abstract}

PACS numbers: $74.20 .-\mathrm{z}, 74.25-\mathrm{q}, 74.40+\mathrm{k}$

The transition from superconductor to insulator (SIT) in the presence of disorder continues to actively attract the interest of experimentalists and theoreticians, alike. Experimentally, this transition has been observed in thinfilms of various materials by several different groups, reviewed for example in [1]. Theoretically, attempts were made to model the original fermionic problem by an effective system of interacting bosons, investigated in depth both analytically and numerically [2,3]. While this approach yields interesting results, it is important to study the fermionic models, which directly correspond to the experimental situation [4]. Recently, fermion models have been studied in the context of the SIT by quantum Monte Carlo (QMC) methods 5 which reproduce the physics accurately. This method yielded the transition from superconducting to insulating behavior as a function of disorder strength. However, several unresolved questions pertaining to the physical origin of this transition remain open. Further, a more microscopic, qualitative understanding of the effects of disorder and the properties of the localized phase would be desirable.

For weak disorder strengths, Anderson's theorem [6] guarantees that the superconducting phase persists despite disorder and the superconducting gap and other thermodynamic properties remain unchanged. The case of stronger disorder and non-uniform order parameters can be treated within the Bogoliubov- de Gennes approach [7]. Such studies have been carried out by several groups [8,9] and the study for s-wave superconductors shows the persistence of a spectral gap with relatively strong disorder [9]. In the spirit of Anderson's theorem, it is expected that the superconducting phase penetrates the localized non-interacting phase until the disorder strength is sufficiently large to overcome the superconducting gap. In this framework, the boundary between the two phases can be estimated from the relation $\Delta \sim \Delta_{1} \sim\left(\nu_{F} l^{d}\right)^{-1}$, where $\Delta$ is the BCS superconducting gap, $\Delta_{1}$ is the level spacing inside a grain of one particle localization length $l$ in the localized non-interacting phase and $\nu_{F}$ is the density of states at the Fermi energy. However, it is not clear whether the mean-field approximation (MFA) remains valid in the presence of medium to strong disorder and its validity is worth testing, by comparisons with QMC calculations. Indeed, an indication of the failure of the MFA comes from recent studies of the Cooper problem for two interacting particles above a frozen Fermi sea, in the three dimensional (3D) Anderson model (incorporating disorder) [10]. This study showed that the attractive Hubbard interaction creates localized pairs in the non-interacting metallic phase, in which the one-particle eigenstates are delocalized. This is in contradiction to the predictions of the MFA according to which Cooper pairs are delocalized in this regime. This disagreement is expected to be even stronger in lower dimensions (namely, the 2D case). Further, the Cooper approximation cannot capture all the many-body effects and this renders interesting the study of the full, interacting, disordered fermion model with finite density of particles.

Contrary to the expectations arising from Anderson's theorem, we find that the attractive interaction that leads to superconductivity gives rise to a phase of bi-particle localized states (BLS) in the metallic regime of the noninteracting disordered model. This confirms the indications obtained from the generalized Cooper problem 10 discussed above. Thus, our results, obtained by exact treatment of all many-body quantum effects in a realistic model at finite particle density, demonstrate convincingly the existence of the BLS phase. Furthermore, we show that the Bogoliubov - de Gennes MFA results are qualitatively incorrect in this phase. The BLS phase is observed at medium disorder strength, while in the limit of weak disorder, the system is superconducting, as expected.

To investigate the interplay of interactions and disorder in the SIT regime, we have studied the attractive Hubbard model with disorder, on a square lattice. The model Hamiltonian reads,

$$
\begin{aligned}
& H=H_{A}+H_{I} \\
& =\left(-t \sum_{\langle i j\rangle, \sigma} \hat{a}_{i, \sigma}^{\dagger} \hat{a}_{j, \sigma}+\sum_{i, \sigma} \epsilon_{i} \hat{a}_{i, \sigma}^{\dagger} \hat{a}_{i, \sigma}\right)+U \sum_{i} \hat{n}_{i \uparrow} \hat{n}_{i \downarrow}
\end{aligned}
$$

where the $\hat{a}_{i, \sigma}^{\dagger}\left(\hat{a}_{i, \sigma}\right)$ are the creation (annihilation) operators for a fermion of spin $\sigma$ at site $i$ with periodic boundary conditions, $\hat{n}_{i \sigma}$ is the number operator for spin $\sigma$ at 
site $i, t$ is the hopping parameter, the Hubbard parameter, $U<0$, gives the strength of the screened attractive interaction and $\epsilon_{i}$, the energy of site $i$ is a random number drawn from a uniform distribution $[-W / 2, W / 2]$, which parameterizes the disorder. The first two terms represent the Anderson Hamiltonian and the last term represents the interaction $H_{I}$. The filling factor $\nu=N_{p} /\left(2 \times L^{2}\right)$, where $N_{p}$ is the number of fermions (particles) and $L$ the linear dimension of the system and the total number of sites is $L^{2}$. It is known that the attractive Hubbard interaction induces superconductivity; thus, we have a useful model without inquiring into the physical origin of the pairing interaction.
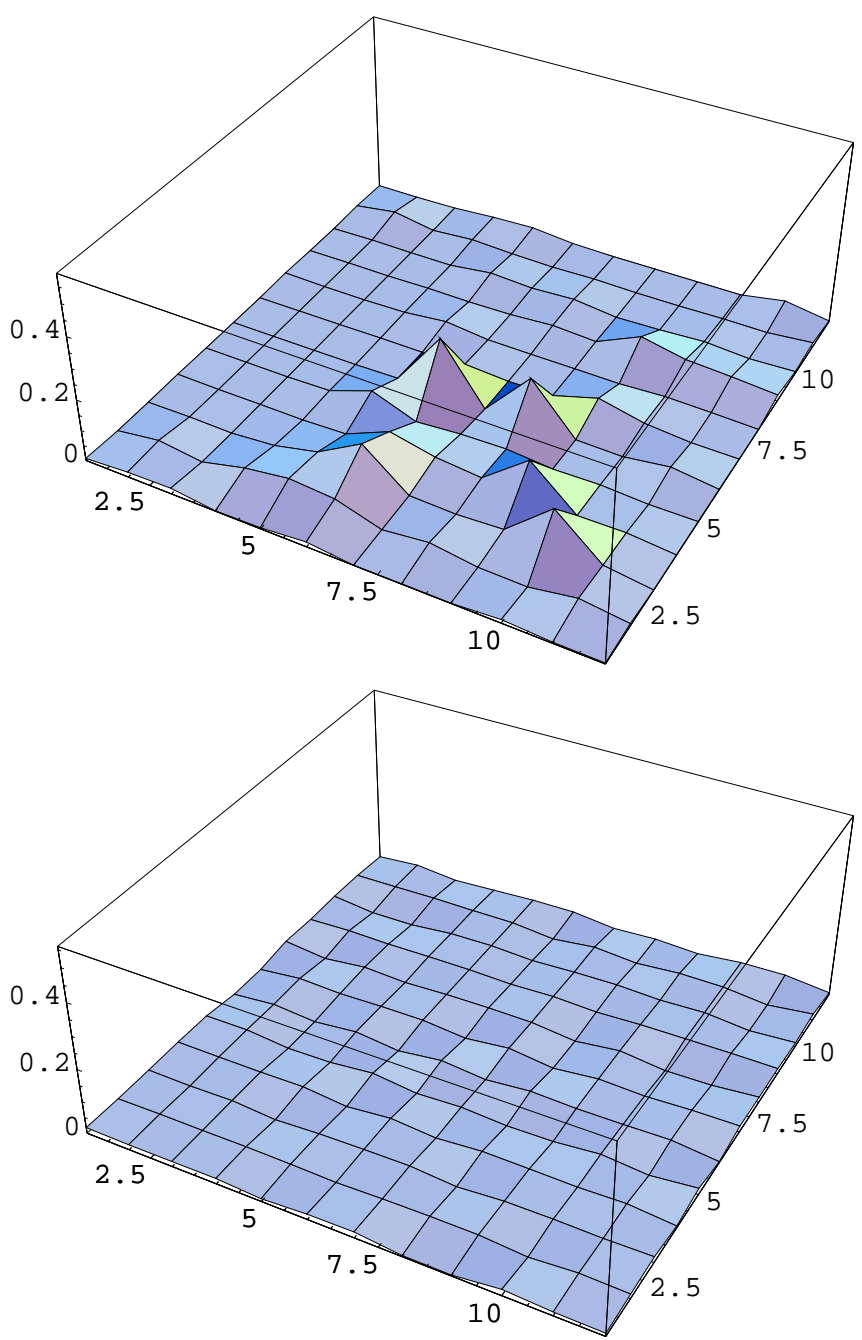

FIG. 1. Charge density difference, $\delta \rho_{i}$ (see text for definition), at sites $i$ of Hamiltonian (1), for a $12 \times 12$ lattice with $W / t=5$ and $N_{p}=72$ fermions for a single disorder realization. The upper part (a) is with $U / t=0$ and the lower part (b) is the result from a Bogoliubov-de Gennes calculation with $U / t=-6.0$.

We have studied this model by the projector quantum Monte Carlo (PQMC) method, which has no fermion sign problem for $U<0$. As is well known [11], this method treats the many-body problem exactly up to statistical errors and gives direct access to the ground state properties of the system. The systematic error arising from the discrete symmetric Trotter decomposition, of step $\Delta \tau$, is of order $(\Delta \tau)^{3}$. The simulations were carried out with $\Delta \tau=0.1$, with up to 60 time steps, in the $S_{z}=0$ sector. The filling factor $\nu$ was tuned around quarter filling, for different disorder and interaction strengths, varying the system size $L$. The largest system studied was 144 sites with 74 particles. We note that this model has been studied in the grand canonical ensemble by a finite temperature QMC method on an $8 \times 8$ lattice with $\nu=0.4375$ [5]. The SIT was estimated in this work to occur at a critical value of disorder strength $W_{c} \approx 3.5 t$. However, the system size dependence of the observables was not investigated in their work.
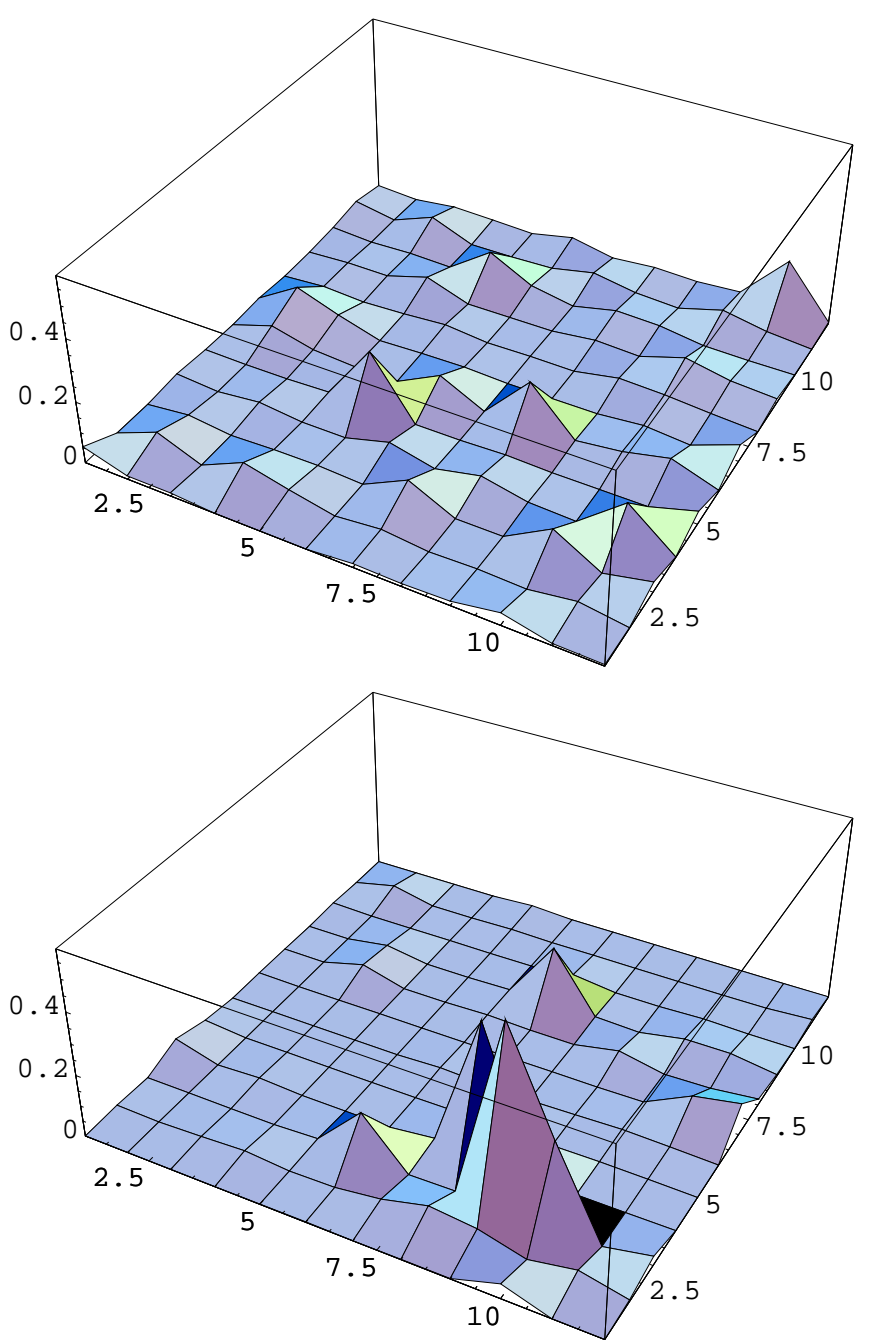

FIG. 2. Charge density difference, $\delta \rho_{i}$ at sites $i$ of Hamiltonian (1), obtained from QMC calculations, shown here for a lattice of $12 \times 12$ sites, with $N_{p}=72$ fermions, for the same disorder realization as in Fig. 1. The upper part (a) is with $W / t=2.0, U / t=-6.0$ and the lower part (b) is with $W / t=5.0$ and $U / t=-6.0$. 
To understand the physics of the model, we consider the difference in charge density in the ground state, $\delta \rho_{i}=$ $\rho_{i}\left(N_{p}+2\right)-\rho_{i}\left(N_{p}\right)$, where $\rho_{i}$ is the charge density at site $i$ and $N_{p}=L^{2} / 2$ corresponds to quarter filling $\left(\sum_{i} \delta \rho_{i}=\right.$ $2)$. For $U=0$, the charge density difference $\delta \rho_{i}$ is given by the one-particle probability $f_{i}=\left|\psi_{i}\right|^{2}$ of the eigenstate at the Fermi level $\left(f_{i}=\delta \rho_{i} / 2\right)$. Due to this relation, the analysis of this characteristic, even in the presence of interactions, allows us to determine whether the charge is localized or delocalized at the Fermi level.

An example of this characteristic for a typical disorder realization is presented in Fig. 1. The results clearly show that the eigenstate at the Fermi level is delocalized in the non-interacting case at the given disorder strength $W / t=5$. Of course, in the limit of $L \rightarrow \infty$, eigenstates are localized for $U=0[12]$. Nevertheless, for the finite system sizes in Fig. 1, the localization length is larger than the system size and the eigenstates correspond to a metallic regime. Certainly, it would be desirable to study this problem in the $3 \mathrm{D}$ case, where the Anderson transition clearly separates the non-interacting delocalized and localized phases. However, it is presently too expensive, numerically, to study comparable system sizes in $3 \mathrm{D}$. In spite of this, our data show that important physical information can be obtained from the $2 \mathrm{D}$ case.

The results obtained from the Bogoliubov-de Gennes MFA for the same disorder realization, with interaction strength $U / t=-6$ are shown in Fig. 1b. These calculations were carried out as described in [8,9]. The eigenvectors and the quasiparticle excitation energies were obtained self-consistently, with good convergence. In addition, we obtained the BCS value for the order-parameter $\Delta \approx 1.36 t$ in the limit of $W / t=0$, at $L=12$ and $U / t=-4$, as in [9]. In the limit $U=0$, this method reproduces $\delta \rho_{i}$ for finite disorder strengths $W / t$. A comparison of Figs. 1a and $1 \mathrm{~b}$ indicates strongly that within the MFA, the charge density at the Fermi level is highly delocalized by the introduction of interactions. This result indicates that interactions smoothen out charge fluctuations within the MFA. Thus, at $W / t=5$ and $U / t=-6$, the MFA gives completely delocalized, metallic behaviour.

The PQMC results for $\delta \rho_{i}$ for the same disorder realization as in Fig. 1 are displayed in Figs. $2 \mathrm{a}$ and $2 \mathrm{~b}$, corresponding to two values of the disorder strength $(W / t=2$ and 5$)$. The model parameters used are identical for Figs. $1 \mathrm{~b}$ and $2 \mathrm{~b}$. It is apparent from Fig. $2 \mathrm{~b}$ that the charge at the Fermi energy is localized. Thus, the PQMC result, with a proper treatment of interactions and disorder, differs qualitatively from the Bogoliubov de Gennes mean-field calculation, which gives the wrong physical picture. We also emphasise that for $U / t=0$, the charge variation $\delta \rho_{i}$ is delocalized (Fig. 1a) and the pronounced, localized peak of charge in Fig. $2 \mathrm{~b}$ is not correlated to the charge distribution of Fig. 1a. With decrease of disorder strength $W$, this localized charge peak seen in Fig. 2b disappears as shown in Fig. 2a. In fact, the charge distribution in Fig. 2a becomes closer to that of the corresponding delocalized, non-interacting case (not shown). These data show that the SIT takes place in the metallic non-interacting phase.

For a more quantitative description of this unusual SIT, we have studied an effective inverse participation ratio (IPR), $\xi$ for an added pair of particles. The IPR is defined as $\xi=\left\langle\sum_{i}\left(\delta \rho_{i} / 2\right)^{2}\right\rangle^{-1}$, where the brackets denote the average over 16 disorder realizations. For $U=0$, this definition exactly reproduces the one-particle IPR at the Fermi level. In the presence of interactions, this quantity allows us to study the charge distribution of added pairs at the Fermi level, beyond the Cooper approximation used in 10. Physically, the IPR gives the number of sites visited by a pair. Thus, the evolution of the IPR with system size $L$, can be used to determine the transition from delocalized to localized behavior.

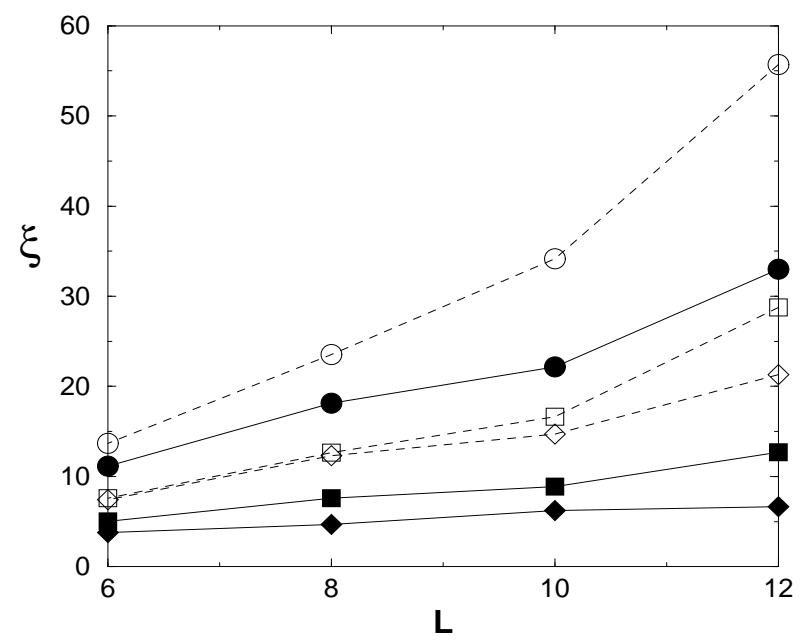

FIG. 3. PQMC result for the IPR $\xi$ obtained from the charge density distribution of the added pair vs. linear dimension of system $L$. Dashed lines are for $W / t=2$ and full lines for $W / t=5$, with $U / t=0$ (circles), -4 (squares) and -6 (diamonds). The average is carried out over 16 disorder realizations.

The PQMC results for the IPR as a function of system size $L$, disorder and interaction strengths are shown in Fig. 3. The data clearly show two main phenomena. Firstly, it is clear that interactions always diminish the IPR, compared to the non-interacting case. Secondly, for moderate disorder strengths the pair becomes localized. Indeed, for $W / t=5, \xi$ continues to grow with $L$ for $U / t=0$ (metallic non-interacting regime), while it remains constant for $U / t=-6$. For example, in the latter case, $\xi=6.7 \pm 0.8$ is much smaller than the total number of sites $\left(L^{2}=144\right)$, clearly indicating pair localization induced by the attractive interaction. This is in great contrast to the MFA result obtained as described for Fig. 1b, which gives complete delocalization with $\xi \approx 124$. In fact, the $\xi$ from the MFA is signifi- 
cantly larger than the non-interacting value of $\xi=33.0$, that once more shows that the MFA gives a physically wrong answer. With the finite system sizes of our simulations, it is difficult to determine the position of the SIT precisely. However, we note that for $W / t=2$, the system remains delocalized even for $U / t=-6$. Thus, we estimate that for $U / t \simeq(-4,-6)$, the critical disorder strength is $W / t \simeq 4$. This is in satisfactory agreement with the results obtained for fixed system size $L=8$ in [5]. We note that, clearly, well below the SIT, at weak disorder, the Bogoliubov - de Gennes theory becomes valid.

In fact, the appearance of this BLS phase can be understood from the following heuristic physical argument [10]. The attractive Hubbard interaction strongly favors pair formation. This effectively doubles the mass of the charge carriers $m^{*} \propto 1 / t$ and thus enhances the effects of disorder induced localization, which are proportion to $W / t \propto m^{*}$. We believe this to be the correct physical reason for the appearance of this BLS phase.

In conclusion, our studies based on the PQMC method show the appearance of a localized phase of pairs which appears in the metallic regime of the non-interacting system. The comparison of these results with Bogoliubov de Gennes mean-field calculations show that the MFA does not predict the existence of this phase. As the non-interacting states are delocalized for the moderate disorder strengths corresponding to the BLS phase, arguments in the spirit of Anderson's theorem should definitely drive the system superconducting at the expense of the non-interacting localized insulating phase. On the contrary, our results provide evidence for the appearance of a new phase which is unexpected from the accepted viewpoint. We expect that this effect exists as well in three dimensions where it should be much more pronounced due to the sharp Anderson transition between the non-interacting metallic and insulating phases.

We thank G. Benenti, G. Caldara, J. Lages and O. Sushkov for useful discussions and remarks and IDRIS, Orsay for access to their supercomputers.

[1] A.M. Goldman and N. Marković, Physics Today 51, November, 39 (1998).

[2] M.P.A. Fisher, G. Grinstein, and S.M. Girvin, Phys. Rev. Lett. 64, 587 (1990).

[3] M. Wallin, E. S. Sørensen, S. M. Girvin, and A. P. Young, Phys. Rev. B 49, 12115 (1994).

[4] A. Larkin, Ann. Phys. (Leipzig) 8, 785 (1999).

[5] R. T. Scalettar, N. Trivedi and C. Huscroft, Phys. Rev. B 59, 4364 (1999).

[6] P. W. Anderson, J. Phys. Chem. Solids, 11, 26 (1959).

[7] P. G. de Gennes, Superconductivity of metals and alloys, (Benjamin, New York 1966).

[8] M. Franz, C. Kallin and A. J. Berlinsky, Phys. Rev. B
54, R6897 (1996).

[9] A. Ghosal, M. Randeria and N. Trivedi, Phys. Rev. Lett. 81, 3940 (1998); cond-mat/0012304.

[10] J. Lages and D. L. Shepelyansky, Phys. Rev. B 62, 8665 (2000).

[11] M. Imada and Y. Hatsugai, J. Phys. Soc. Jpn. 58, 3752 (1989); B. Srinivasan, S. Ramasesha and H. R. Krishnamurthy, Phys. Rev. B 541692 (1996).

[12] E. Abrahams, P. W. Anderson, D. C. Licciardello, and T. V. Ramakrishnan, Phys. Rev. Lett. 42, 673 (1979). 